Advances in Radio Science (2003) 1: 309-311

(C) Copernicus $\mathrm{GmbH} 2003$

\title{
Scattering in remote sensing in the visible and microwave spectral range and in traffic control
}

\author{
U. Böttger, R. Kühne, and K.-U. Thiessenhusen \\ Deutsches Zentrum für Luft- und Raumfahrt, Institut für Verkehrsforschung, Rutherfordstrasse 2, 12489 Berlin, Germany
}

\begin{abstract}
The treatment of scattering processes in remote sensing for interpretation of satellite data is demonstrated in the visible and microwave spectral range comparing the two spectral ranges. Analogies and distinctions in the treatment of the scattering processes are shown. Based on this cognition an approach for traffic simulation is outlined.

Simulating the traffic of a part of a city, a whole city or a larger area in an acceptable time is one of the tasks in recent traffic research. One possible approach is the areal treatment of the road network. That means that single streets are not resolved but are introduced into simulations only by parameters that correspond to a specific traffic area resistance. The aim of this work is to outline such a possibility using experiences obtained from the theory of radiative transport to simulate scattering processes and applying them to the very complex system of traffic simulation.
\end{abstract}

\section{Radiative transfer and scattering}

Radiative transfer theory (Chandrasekhar, 1950) describes the scattering and absorption processes in remote sensing from the ultraviolet and visible to the microwave spectral range. It describes the change a radiation field experiences traversing through a medium. Starting point of this theory is the radiative transfer equation in the general case.

$\Omega \nabla \boldsymbol{I}_{\lambda}=-\mathbf{k}_{\lambda} \boldsymbol{I}_{\lambda}+\mathbf{k}_{\lambda} \boldsymbol{J}_{\lambda}$

$\boldsymbol{I}_{\lambda}$ is the intensity or the Stokes vector depending on wavelength $\lambda . \Omega$ is the solid angle. $\mathbf{k}_{\lambda}$ is the extinction coefficient matrix. $\boldsymbol{J}_{\lambda}$ is called the source function. The first term on the right hand side corresponds to the reduction of $\boldsymbol{I}_{\lambda}$, which is caused by absorption of the medium and by scattering in the medium. The second term implies the enhancement of $\boldsymbol{I}_{\lambda}$, which arises from emission and scattering from all directions into the direction of observation. The different terms of

Correspondence to: U. Böttger

(ute.boettger@dlr.de) the radiative transfer equation are modified and specified in dependence on the observed system.

For a plane-parallel atmosphere surface system the equation of transfer is

$$
\begin{aligned}
& \frac{d \boldsymbol{I}(\mathbf{n}, \lambda, T)}{d s}=-\mathbf{k}(\mathbf{n}, \lambda) \boldsymbol{I}(\mathbf{n}, \lambda, T) \\
& +\int_{4 \pi} \mathbf{p}\left(\mathbf{n}, \mathbf{n}^{\prime}, \lambda\right) \boldsymbol{I}\left(\mathbf{n}^{\prime}, \lambda, T\right) d \mathbf{n}^{\prime}+\mathbf{a}(\mathbf{n}, \lambda) B(\lambda, T)
\end{aligned}
$$

where $\mathbf{n}$ is the vector of direction of propagation. $\mathbf{a}(\mathbf{n}, \lambda)$ is the absorption coefficient vector, which includes the absorption of atmospheric gases and the absorption by aerosol and cloud particles. $T$ is the temperature necessary to calculate the Planck function $B(\lambda, T)$. The scattering function $\mathbf{p}\left(\mathbf{n}, \mathbf{n}^{\prime}, \lambda\right)$ describes the probability, that the intensity from direction $\mathbf{n}^{\prime}$ is scattered in direction $\mathbf{n}$ (Fig. 1). It is a function of the physical properties (refractive index) and of the size distribution of the scattering particles.

The effect of the different terms in Eq. (2) depends on the considered wavelength range. In the ultraviolet, visible and near infrared spectral range the incident sun light is scattered and absorbed in the atmosphere. This is described by the first two terms on the right hand side in the equation. The second term contains the direct scattered sun light and the multiple scattered light in the atmosphere. Scattering at air molecules and at very small particles, the so called Rayleigh scattering, has its largest effect in the ultraviolet. In the visible and near infrared spectral range the scattering at aerosol and cloud particles gets a larger influence compared to Rayleigh scattering. The influence of these particles decreases with increasing wavelength. In the microwave and millimeter wavelength range only very large particles (e.g. rain drops) remain important. The intensity of the incident solar radiation decreases with increasing wavelength as well and the influence of the third term in Eq. (2) increases, which describes the thermal emission as a function of the temperature.

Several approaches exist to solve the radiative transfer equation for an inhomogeneous atmosphere. All approaches 


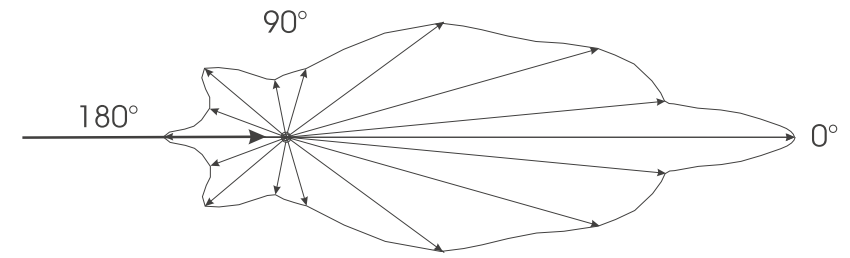

Fig. 1. Schematic representation of the distribution of intensity scattered at a particle. The intensity distribution shows strong forward scattering $\left(0^{\circ}\right)$, less backward scattering $\left(180^{\circ}\right)$ and a small scattering peak at $140^{\circ}$ scattering angle.

divide the inhomogeneous atmosphere in homogeneous layers or cells, for which the transfer equation is solved.

A layer is considered homogeneous, if the extinction matrix, the absorption coefficient vector and the scattering function are representative and constant for this layer. The solution of the radiative transfer equation for the whole atmosphere surface system is derived from the solutions of the homogeneous layers (Fig. 2) applying the interaction principle (Plass et al., 1973). To link the layers it is assumed that the radiation emerging from one layer or cell is entering into the next layer or cell without changing. In the next layer or cell it is then altered by scattering, absorption, transmission and emission corresponding to the physical characteristics of the layer or cell. There are several methods and procedures available to solve the radiative transfer equation for a homogeneous layer (Lenoble, 1985) and to link the homogeneous layers, which will not be considered here.

\section{Application to traffic simulation}

The steadily growing interest in understanding and improving the traffic situation makes it necessary to find new approaches for analysis and simulation of traffic flow. In this paper it is investigated, if approaches to solve the radiative transfer problem in remote sensing can be adopted to traffic simulation. Comparing traffic simulation with the treatment of scattering processes in an atmosphere-surface-system the similarities are obvious. The radiative transfer problem describes transmission, distribution and quantitative change of a radiation field incident on a medium. In traffic simulation traversing, distribution by change of direction and quantitative change of traffic flow in an area are described. The problem can be treated in both cases as a stationary problem if the considered time frame is chosen large enough. The distribution of radiation is described by the scattering function. For traffic simulation an appropriate quantity can be found and defined. Traffic flow, destination and origins of traffic correspond to transmission, absorption and emission processes in radiative transfer.

The medium of propagation in radiative transfer is the atmosphere or the ocean. The medium of propagation in traffic simulation is the network of roads in a certain area. We consider here the network of roads not on the level of single

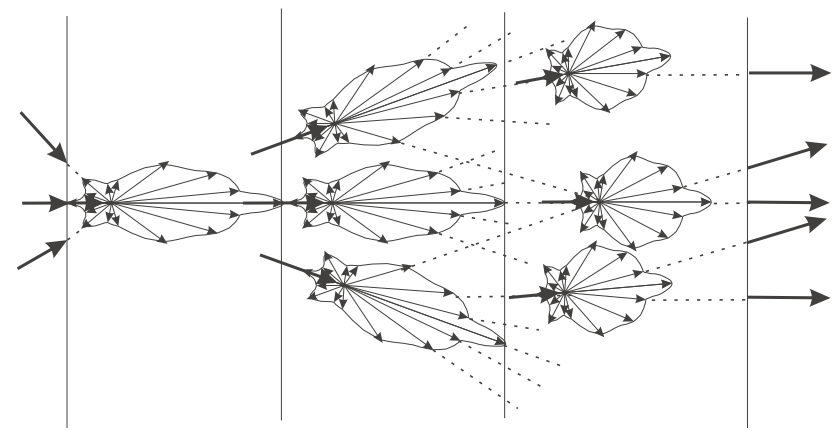

Fig. 2. Schematic illustration of radiation traversing through a multilayered system. The system is subdivided in homogeneous sublayers (between two vertical lines), that are characterized by constant scattering function, extinction and scattering parameters.

roads but as a pattern of cells. The cells are characterized by parameters describing the redistribution of traffic inside the cell. After the cells can be linked as described for the linking of layers in radiative transfer simulation.

Figure 3, upper part shows an example for a typical traffic situation in the city of Vienna, Austria. Here, the averaged speed in quadratic cells of $100 \mathrm{~m} \times 100 \mathrm{~m}$ is displayed color coded. In bright areas, the averaged speed is large. The velocities are calculated using Floating Car Data from a Vienna taxi fleet. Figure 3, lower part shows that by simple abstraction a cell system can be deduced. To this cell system the radiative transfer approach can be applied.

There are obvious analogies between radiative transfer and this way of describing traffic. The concept of application of methods for radiative transfer simulation to traffic simulation has the following view: An area is subdivided corresponding to traffic characteristics into small cells considered as homogeneous. These characteristics can be the change of the speed and direction and distribution of traffic in the cell, number of vehicles, that are absorbed by or emitted from the cell. In these cells the traffic from all directions into this cell is changed by applying these characteristics like an operator to the incoming traffic flow. The traffic changed in this way leaves the cell then again in all direction according to its modification. The linking between the cells takes place as mentioned above.

In both cases, it is possible to retrieve the situation within a certain area by solving an inverse problem. The input for the inverse problem is derived from measurements of the characteristics at a few distinct points outside the area of interest.

But the differences between the two approaches are obvious as well. The considered radiation field is continuous. Traffic has discrete characteristics, even if it is considered as continuous. Thus the distribution function is cascaded. The symmetry relations, that are important in radiative transfer, have to be neglected in traffic simulation. This leads to the effect, that the scattering function in radiative transfer is valid for an angle range between $0^{\circ}$ and $180^{\circ}$, but in traffic simulation angles larger that $180^{\circ}$ must be taken into account. But the problems arising because of these differences are solv- 

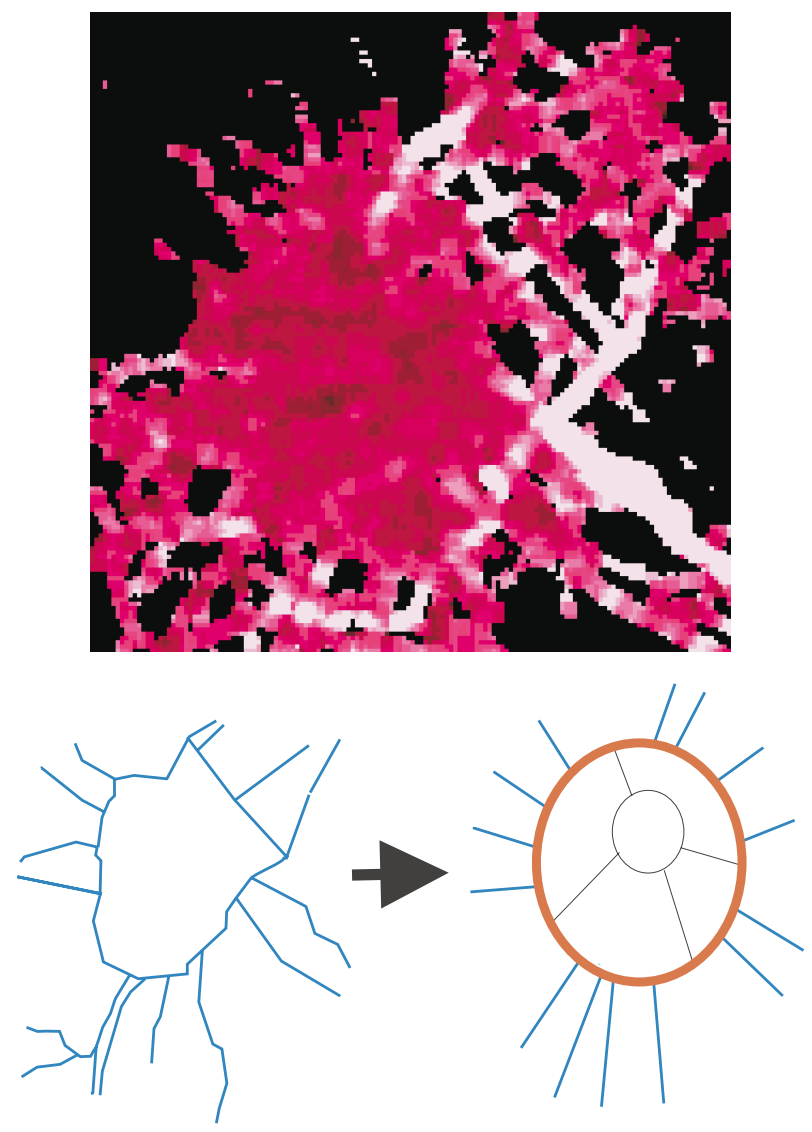

Fig. 3. (a) Illustration of typical traffic situation in the city area of Vienna. The speed derived from Floating Car Data averaged over cells of $100 \mathrm{~m} \times 100 \mathrm{~m}$ is displayed color coded. Dark red areas correspond to low velocity, bright areas correspond to high velocity. In black areas, there are no important roads. (b) By simple abstraction a layer or cell system can be deduced. able. It is much more difficult to provide the quantities corresponding to scattering, absorption or emission in radiative transfer. Solving these difficulties the well studied retrieval methods in radiative transfer can be applied to traffic simulations. In this way the traffic situation in the homogeneous cells can be derived from few traffic flow measurements at distinct points, that are indicators of the traffic situation inside the area of interest.

Acknowledgements. The authors wish to thank Ralf-Peter Schäfer (DLR Berlin), WIHUP-Taxi (Vienna) and Austrosoft (Vienna) for supply of the floating car data.

\section{References}

Chandrasekhar, S.: Radiative Transfer, Oxford University Press, 1950.

Plass, G. N., Kattawar, G. W., and Catchings F. E.: Matrix operator theory of radiative transfer. 1: Rayleigh scattering, 12, 314-329, 1973.

Lenoble, J.: Radiative Transfer in Scattering and Absorbing Atmospheres: Standard Computational Procedures, A. Deepak Publ., Hampton, Virginia, USA, 1985. 